\title{
Path Following for an Articulated Vehicle Based on Switching Model Predictive Control Under Varying Speeds and Slip Angles
}

\author{
Thaker Nayl \\ thanay@1tu.se \\ George Nikolakopoulos \\ geonik@ltu.se \\ Thomas Gustafsson \\ tgu@ltu.se \\ Control Engineering Group \\ Department of Computer Science, Electrical and Space Engineering \\ Luleå University of Technology \\ Luleå, Sweden, SE-97187.
}

\begin{abstract}
This article is focusing on the problem of path following for an articulated vehicle under varying velocities and slip conditions. The proposed control architecture consists of a switching control scheme based on multiple model predictive controllers, fine tuned for dealing with different operating speeds and slip angles. In the presented analysis for the non-holonomic articulated vehicle, the corresponding kinematic model is being transformed into an error dynamics model, which is linearized around multiple nominal slip angle cases and various operating speeds. The existence of the slipping and varying speed has a significant effect on the vehicle's path following capability and can significantly deteriorate the performance of the overall control scheme. Based on the derived multiple dynamics modeling, the current slip and vehicle's speed are being considered as the signal selector for the proposed switching model predictive control scheme. The efficacy of the proposed controller is being evaluated by an extended set of simulation results.
\end{abstract}

\section{Introduction}

In general an articulated vehicle consists of two parts, a front and a rear, linked with a rigid free joint. Each body has a single axle and the wheels are all non-steerable, while the steering action is being performed on the joint, by changing the corresponding articulated angle, between the front and the rear of the vehicle.

In the relative literature, there have been several research approaches for the problem of modeling articulated vehicles, based on the theory of multiple body dynamics $[7,11,13]$. Most of these methods contain simple models that: a) are not taking under consideration the effect of the slip angles, as the complexity of the overall problem is being increased and it is more difficult to proceed to the next stage of the control scheme design based on highly non-linear articulated vehicle's dynamics, while b) in these modeling approaches, speed has been considered as a constant, an assumption that it is not globally valid, while at the same time the speed level is also effecting the slippage that the vehicle is being experiencing.

As slip angle is one of the most important factor that degrades the overall performance of the articulated vehicle, various efforts have been proposed until now for estimating or measuring these effects $[12,15]$, while still the existence of full kinematic models that will integrate the slip angles, for the case of articulated vehicles, is obsolete. From a control point of view, there have been proposed many traditional techniques for non-holonomic vehicles, based on error dynamics models without the presence of slip angles. More analytically, in [11] linear control feedback has been applied, while in [12] a Lyapunov based approach has been presented. In [2] a control scheme based on linear matrix inequalities has been presented and in [14] a pole placement technique has been applied. Moreover, in [5] the authors have presented a path tracking controller based on error dynamics, while in [1] the general problem of designing a path following controller for a $n$-rear vehicle, based on non-linear adaptive control has been derived. Finally, classical fundamental problems of motion control for articulated vehicles have been presented in $[4,7]$ and [3].

The novelty of the proposed article stems from: a) the derivation of an error dynamics modeling framework for the case of an articulated vehicle operating under varying speeds and slip angles, and b) adopting a switching model control scheme that is able to take under consideration the effects of real life constraints on the control input (articulated angle) and mechanical restrictions, while at the same time contains multiple switching model predictive controllers that are being fine tuned to optimize path tracking under varying slip angles and speeds. The overall resulting control scheme is ensuring smooth transition of the control effort as the articulated vehicle is being driven with different speeds, over regions of different slippage, while retaining the stability of the articulated vehicle. 


\section{Articulated Vehicle Modeling}

\subsection{Kinematic Model Without Slip Angles}

First the case of an articulated vehicle, under no effect of slip angles $(\alpha=\beta=0)$ is being presented, as it is depicted in Figure 1, where $\left(x_{f}, y_{f}\right)$ and $\left(x_{r}, y_{r}\right)$ denote the coordinates of the front and the rear, $p_{f}$ and $p_{r}$ are the corresponding centers of gravity, $l_{f}$ and $l_{r}$ are the lengths of the front and rear, while the angles $\theta_{f}$ and $\theta_{r}$ denote the vehicle's part orientation. The $(x, y)$ axes represent the fixed coordinating system, defined as [11]. By examining the vehicle's depicted geometry, as also the relation between the coordinates of $p_{f}$ and $p_{r}$, it can be extracted that:

$$
\begin{aligned}
& \dot{x}_{f}=v_{f} \cos \theta_{f} \\
& \dot{y}_{f}=v_{f} \sin \theta_{f}
\end{aligned}
$$

The velocities at the front and the rear parts have the same changing with respect to the velocity at the rigid free joint of the vehicle, while the relative velocity vector equations can be defined as:

$$
\begin{aligned}
v_{f} & =v_{r} \cos \gamma+\dot{\theta}_{r} l_{r} \sin \gamma \\
v_{r} \sin \gamma & =\dot{\theta}_{f} l_{f}+\dot{\theta}_{r} l_{r} \cos \gamma
\end{aligned}
$$

Where $v_{f}$ and $v_{r}$ are the velocities of the front and rear parts respectively, the articulated angle $\gamma$ is being defined as the difference between the orientation angles $\theta_{f}$ of the front and $\theta_{r}$ of the rear part of the vehicle. By combining the above equations using the relation $\theta_{r}=\theta_{f}-\gamma$, the angular velocity $\dot{\theta}_{f}$ of the front is being calculated as:

$$
\dot{\theta}_{f}=\frac{v_{f} \sin \gamma+l_{r} \dot{\gamma}}{l_{f} \cos \gamma+l_{r}}
$$

The corresponding angular velocities for the front and rear parts, which are being defined as $\dot{\theta}_{f}$ and $\dot{\theta}_{r}$ respectively, have different values when $\left(l_{f} \neq l_{r}\right)$ or the vehicle is not driving straight $(\gamma \neq 0)$. Finally, the angular velocity of the rear is being defined as $\dot{\theta}_{r}=\dot{\theta}_{f}-\dot{\gamma}$, or:

$$
\dot{\theta}_{r}=\frac{v_{f} \sin \gamma-l_{f} \dot{\gamma} \cos \gamma}{l_{f} \cos \gamma+l_{r}}
$$

\subsection{Kinematic Model Under Slip Angles}

In the case of slip angles, the kinematic model of the articulated vehicle can be also formulated from general geometry that includes ideal and slip behavior for the steering angles. The definition of this model has been initially based on the derivation in [15], which included the perturbed factors in the vehicle position as two slip variables $\beta$ and $\alpha$, being defined as the front's and rear's slip angles respectively. Under the influence of slip angles, the vehicle's configuration is depicted in Figure 1, where $r_{1}, r_{2}$ are the radiuses from instantaneous centers of velocity for the front and the rear respectively. For the initial center curvature of the trajectory it is being assumed that the vehicle is

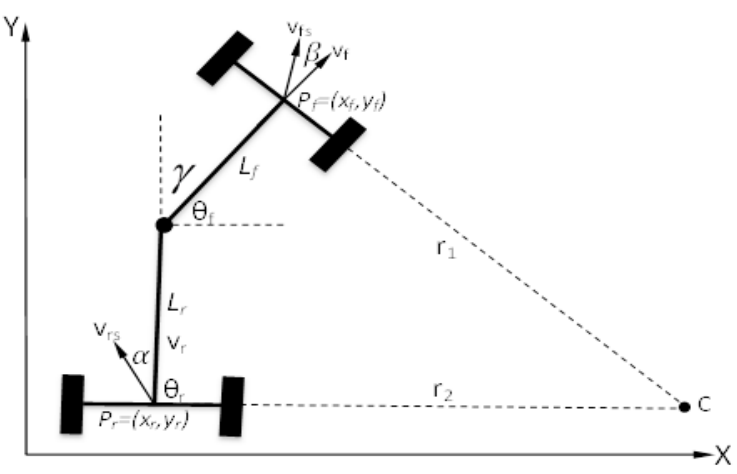

Figure 1. Articulated vehicle modeling con-
figuration under the influence of slip angles

moving forward without slip conditions. In the following derivation, the unit subscript 's' denotes variables in the slip angle case as they have been defined previously in the non-slip examined case. The kinematic equations, for the motion under the effect of slip angles, for the front part can be formulated as:

$$
\begin{aligned}
& \dot{x}_{f s}=v_{f} \cos \left(\theta_{f}+\beta\right) \\
& \dot{y}_{f s}=v_{f} \sin \left(\theta_{f}+\beta\right)
\end{aligned}
$$

In this case, the vehicle's motion depends not only on vehicle's velocities, the articulated angle and the vehicle's lengths, but also on the slip angles, while the resulting vehicle's heading is provided by a combination of the slip angles with the orientation angles. The rate of the orientation $\dot{\theta}_{r s}$ can be defined as function of the steering angle $\gamma$ and both slip angles. Based on the assumption that the vehicle develops a steady-state motion turning, this rate can be provided by the utilization of a virtual center of rotation, depending on the velocity [8]. In the examined case, the front's and rear's speeds can be computed as:

$$
\begin{array}{r}
v_{f} \cos \alpha=v_{r} \cos (\gamma-\alpha)+\dot{\theta}_{r} l_{r} \sin (\gamma+\beta-\alpha) \\
v_{r} \sin (\gamma+\beta-\alpha)=\dot{\theta}_{f} l_{f} \cos \alpha+\dot{\theta}_{r} l_{r} \cos (\gamma-\alpha)
\end{array}
$$

while, by solving the above equations, the angular velocities for the front and the rear can be defined as:

$$
\begin{aligned}
\dot{\theta}_{f s} & =\frac{v_{f} \sin (\gamma+\beta-\alpha)+l_{r} \dot{\gamma} \cos \alpha}{l_{f} \cos (\gamma-\alpha)+l_{r} \cos \alpha} \\
\dot{\theta}_{r s} & =\frac{v_{f} \sin (\gamma+\beta-\alpha)-l_{f} \dot{\gamma} \cos (\gamma-\alpha)}{l_{f} \cos (\gamma-\alpha)+l_{r} \cos \alpha}
\end{aligned}
$$

It should be noted that equations $(11,12)$ are more accurate than equations $(7,8)$, if the slip angles are known a priori, a task that is quite difficult as the slip angles are dependent of the vehicle's velocity, mass, tire-terrain interaction and articulation angle, in a highly non-linear way. 


\section{Error Dynamics Modeling}

Based on the assumption that the vehicle develops a steady-state motion turning, the rates of the orientation change are being provided by: $r_{1}=\frac{v_{f}}{\dot{\theta}_{f}}$ and $r_{2}=\frac{v_{r}}{\dot{\theta}_{r}}$. In Figure 2 it is depicted an overview of how the displacement, heading and curvature errors are being defined, between the actual path of the articulated vehicle and the desired one, under the effects of slipping. The distance from the vehicle to the reference path displacement, the angle between the vehicle and the reference are also being displayed.

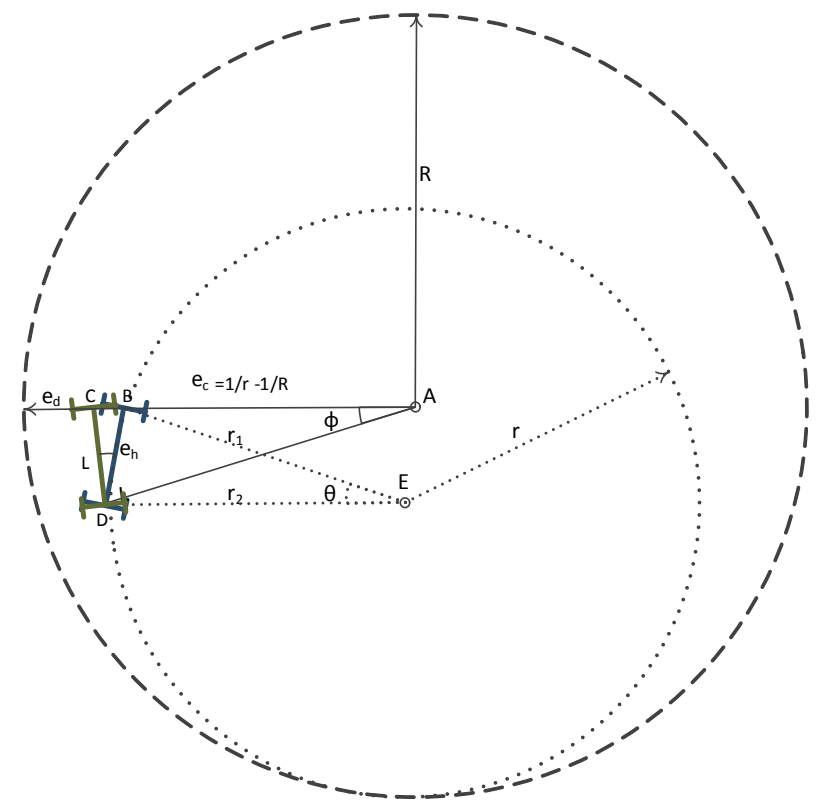

\section{Figure 2. Articulated vehicle following error transformation}

Based on [13], in the following derivation, three errors under the effects of slip angles are being defined: a) $e_{d}$ is the displacement error, b) $e_{h}$ is the heading error, and c) $e_{c}$ is the curvature error. The displacement error $e_{d}$ is the difference between the coordinates of the front and the coordinates of the desired circular path. From the triangle $(A B D)$, if it is assumed that $\phi$ and $\theta$ are small, and can be defined that: $\theta \triangleq\left(\frac{l_{f}+l_{r} \cos (\gamma+\beta)}{r}\right)$ and from the triangle $(D B C), e_{h} \triangleq \frac{e_{d}}{l_{f}}$, while the error in the displacement is: $e_{d}=e_{h} r \theta-l_{f} \cos (\gamma+\beta)$, and by taking the time derivative of this equation it is derived that:

$$
\dot{e}_{d}=v e_{h}+\dot{\gamma} l_{f} \sin (\gamma+\beta)
$$

The heading error $e_{h}$ is the orientation difference between the centers of the vehicle and the circular path. A change in the heading error is being defined as: $e_{h} \triangleq(\theta-\phi)+$ $(\gamma \sin (\gamma+\beta-\alpha))$, and it is being assumed that: $\theta r \bumpeq \phi R$. From this equation it can be derived that $e_{h}=\theta\left(1-\frac{r}{R}\right)+$ $\left(\gamma \sin (\gamma+\beta-\alpha)\right.$. The curvature error $e_{c}$ measures the difference between the vehicle's paths and the curvature of the trajectory path is: $e_{c}=\frac{1}{r}-\frac{1}{R}$. By utilizing these relations, the curvature error is being defined as:

$$
\dot{e}_{h}=v e_{c}+\dot{\gamma} \sin (\gamma+\beta-\alpha)+\dot{\gamma} \gamma \cos (\gamma+\beta-\alpha)
$$

With the assumption that the slip angles, the velocity and the curvature of the trajectory are piecewise constant and by differentiating the equation: $e_{c}=\frac{v \sin (\gamma+\beta-\alpha)+l_{r} \dot{\gamma} \cos \alpha}{v\left(l_{f} \cos (\gamma-\alpha)+l_{r} \cos \alpha\right)}$ with respect to time, the rate of the curvature error is being defined as:

$$
\begin{aligned}
\dot{e}_{c}=\quad & \quad \dot{\gamma} \frac{l_{f} \cos (\beta)+l_{r} \cos (\alpha) \cos (\gamma+\beta-\alpha)}{\left(l_{f} \cos (\gamma-\alpha)+l_{r} \cos (\alpha)\right)^{2}}+ \\
& \ddot{\gamma} \frac{l_{r}\left(l_{f} \cos (\gamma-\alpha) \cos (\alpha)+l_{r} \cos (\alpha)^{2}\right)}{v\left(l_{f} \cos (\gamma-\alpha)+l_{r} \cos (\alpha)\right)^{2}}+ \\
& \dot{\gamma}^{2} \frac{l_{f} l_{r} \sin (\gamma-\alpha) \cos (\alpha)}{v\left(l_{f} \cos (\gamma-\alpha)+l_{r} \cos (\alpha)\right)^{2}}
\end{aligned}
$$

Linearizing the error dynamics in the equations (13), (14) and (15) around the reference path with constant articulated and slip angles under small displacement, yields:

$$
\begin{gathered}
\dot{e}_{d}=v e_{h}+\dot{\gamma} l_{f} \beta \\
\dot{e}_{h}=v e_{c}+\dot{\gamma}(\beta-\alpha) \\
\dot{e}_{c}=\dot{\gamma} \frac{1}{\left(l_{f}+l_{r}\right)}+\ddot{\gamma} \frac{l_{r}}{v\left(l_{f}+l_{r}\right)}+\dot{\gamma}^{2} \frac{l_{f} l_{r}(\gamma-\alpha)}{v\left(l_{f}+l_{r}\right)^{2}}
\end{gathered}
$$

where it can be observed that $\dot{e_{c}}$ is dependant on the slippage and the rate of change of articulation angles. By performing the following change of variable:

$$
\dot{e}_{c}=\dot{e}_{c}-\dot{\gamma} \frac{l_{r}}{v\left(l_{f}+l_{r}\right)}
$$

The following state space error dynamics description for the articulated vehicle is being extracted:

$$
\left[\begin{array}{c}
\dot{e}_{d} \\
\dot{e}_{h} \\
\dot{e}_{c}
\end{array}\right]=\left[\begin{array}{lll}
0 & v & 0 \\
0 & 0 & v \\
0 & 0 & 0
\end{array}\right]\left[\begin{array}{c}
e_{d} \\
e_{h} \\
\dot{e}_{c}
\end{array}\right]+\left[\begin{array}{c}
l_{f} \beta \\
\frac{l_{r}+(\beta-\alpha)\left(l_{f}+l_{r}\right)}{\left(l_{f}+l_{r}\right)} \\
\frac{1}{\left(l_{f}+l_{r}\right)}
\end{array}\right] \dot{\gamma}
$$

In the case that the articulated vehicle is being driven with varying speed, over terrains that are characterized by different slip angles, multiple piecewise operating sets for the speed and the slip angles, of the same characteristics, can be defined as: $v_{i} \in \mathscr{L}_{1}, \beta_{i} \in \mathscr{L}_{2}$ and $\alpha_{i} \in \mathscr{L}_{3}$, with $\mathscr{L}_{1}, \mathscr{L}_{2} \mathscr{L}_{3} \in \mathfrak{R}^{3}$ and $i \in \mathbf{S}^{+}$, can be defined, leading to a switching state space description for the model as it follows:

$$
\dot{\mathbf{x}}=\mathbf{A}_{i} \mathbf{x}+\mathbf{B}_{i} \dot{\gamma}
$$

$\mathbf{x} \in \mathscr{X} \subseteq \mathfrak{R}^{3}$ is the state vector, $\dot{\gamma} \in \mathscr{U} \in \mathfrak{R}$ is the control action, $\overline{\mathbf{A}}_{\mathbf{i}} \in \mathfrak{R}^{3 \times 3}, \mathbf{B}_{\mathbf{i}} \in \mathfrak{R}^{3 \times 1}$, and full state feedback is being considered, or $\mathbf{C}=\mathbf{I}_{3 \times 3}$. The variables $\gamma$ and $\dot{\gamma}$ can be measured with a great accuracy, while an estimation algorithm or a look up table should be utilized for determining the value of the slip parameters. 


\section{Model Predictive Control Design}

MP-controller has been very successful in practice and its a highly effective control scheme that is able to take under consideration multiplicative system model descriptions, uncertainties, nonlinearities and physical and mechanical constraints in the system model parameters or in the control signals [10]. In the current research effort, the MP-controller schemes are able to predict future values of the vehicles error dynamics based on the present available information and the current constraints [6]. The MPcontroller action, which is the rate of articulation angle, is based on a finite horizon continue time minimization of predicted tracking error with constraints on the control inputs and the state variables. The overall block diagram of the proposed closed loop system is depicted in Figure 3. For efficiently controlling the articulated vehicle, the controller utilizes the current state of motion of the vehicle as well as the next target points of the reference trajectory. The path planner is generating the desired path, while in the sequel this path (planar coordinates) is being translated to displacement, heading and curvature coordinates that act as the reference input for the MP-controller.

The formulation of the MP-controller is based on: a) the current full state feedback, b) the active constrains on the system, c) the estimated slip angles, d) the measured velocity and e) the mode selector signal, which depends on the slipping and the velocity of the vehicle, in order to apply switching optimal control action.

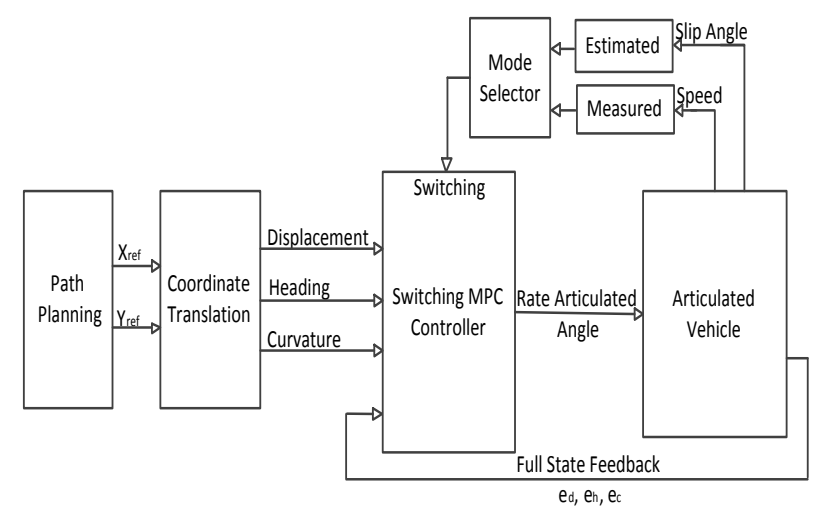

Figure 3. Switching MP-controller scheme
block diagram

The construction of the MP-controller is based on the system description defined in equation (21). The mode selector signal $i \in \mathscr{S}$ with $\mathscr{S} \triangleq\{1,2, \cdots, s\}$ is a finite set of indexes and $s$ denotes the number of switching subsystems in (24). For polytypic description, $\Sigma$ is the polytope $\Sigma$ : $\operatorname{Co}\left\{\left[\mathbf{A}_{i} \mathbf{B}_{\mathbf{i}}\right], \cdots,\left[\mathbf{A}_{i} \mathbf{B}_{\mathbf{s}}\right]\right\}$, Co denotes the convex hull and $\left[\mathbf{A}_{i}, \mathbf{B}_{\mathbf{i}}\right]$ are the vertices of the convex hull. Any $\left[\mathbf{A}_{\mathbf{i}}, \mathbf{B}_{\mathbf{i}}\right]$ within the convex set $\Sigma$ is a linear combination of the vertices $\sum_{j=1}^{s} \mu_{j}\left[\mathbf{A}_{\mathbf{i}} \mathbf{B}_{\mathbf{i}}\right]$ with $\sum_{j=1}^{s} \mu_{j}=1,0 \leq \mu_{j} \leq 1$.

In the presented methodology for the design of the
MP-controller scheme, only the front slip angle $\beta$ has been taken under consideration as it has the more significant effect on the behavior of the vehicle than the rear slip angle $\alpha$, while at the same time $\alpha$ has the opposite sign of $\beta$. As a result of this remark the mode selector signals of the MPC are the measured velocity $v_{i}$ of the vehicle and the estimated slip angle $\beta_{i}$. For defining the switching instances, the sets $\mathscr{L}_{1}$, and $\mathscr{L}_{2}$ have been discretized into operating subspaces. The discretization of the speed and slip angle operating sets, can be formulated by defining multiple nominal values $v_{0}, \beta_{0}$ and allowing them to take values into neighboring regions of lengths $\xi_{i}$ and $\psi_{i}$. This can be mathematically being formulated as:

$$
\begin{aligned}
& \mathscr{L}_{1, i}=v_{0, i}^{\min }=v_{0, i}-\xi_{i} \leq v_{0, i} \leq v_{0, i}+\xi_{i}=v_{0, i}^{\max } \\
& \mathscr{L}_{2, i}=\beta_{0, i}^{\min }=\beta_{0, i}-\psi_{i} \leq \beta_{0, i} \leq \beta_{0, i}+\psi_{i}=\beta_{0, i}^{\max }
\end{aligned}
$$

The sets $\mathscr{X}$ and $\mathscr{U}$ specify state and input constraints. Let the set $\mathscr{X}$ contain the $\mathbf{x}$ states that satisfy the following bounding inequality:

$$
\mathbf{x}^{\min }=\mathbf{x}-\Delta_{1} \leq \mathbf{x} \leq \mathbf{x}+\Delta_{1}=\mathbf{x}^{\max }
$$

where $\Delta_{1} \in \mathfrak{R}_{+}^{(3,1)}$ is the vector containing the selecting state boundary conditions. The control input bounding set $\mathscr{U}$ can be derived by taking under consideration the mechanical and the physical constraints of the articulated vehicle, as also the preference on aggressive or not maneuvers. These constraints can be also formulated as presented in (23).

$$
u^{\min }=u-\Delta_{2} \leq u \leq u+\Delta_{2}=u^{\max }
$$

where $\Delta_{2} \in \Re_{+}$is the vector containing the selecting control boundary conditions. Let the matrix $\mathbf{H}_{\mathbf{i}}$ be a zeroed $2 \times 2$ matrix with its $i-$ th column equal to $[1,-1]^{T}$, and the other $\mathbf{0}_{2,1}$ i.e. for $i=2$ :

$$
\mathbf{H}_{\mathbf{i}}=\left[\begin{array}{cc}
0 & 1 \\
0 & -1
\end{array}\right]
$$

Then the previous bounds in (22) and (23) can be cast in a more compact form as:

$$
\left[\begin{array}{l}
\mathbf{H}_{1} \\
\mathbf{H}_{2}
\end{array}\right]_{4 \times 2} \cdot\left[\begin{array}{c}
\mathbf{x} \\
-- \\
u
\end{array}\right]_{2 \times 1} \leq\left[\begin{array}{c}
\mathbf{x}^{\max }+\Delta_{1} \\
----- \\
u^{\max }+\Delta_{2}
\end{array}\right]_{4 \times 1}
$$

These constraints are embedded in the MP-controller computation algorithm in order to compute an optimal controller that counts for the physical and mechanical constraints that restrict the articulated vehicle's motion.

The basic idea of MP-controller is to calculate a sequence of future control actions in such a way that it minimizes a cost function defined over a predefined prediction horizon. More specifically, the $(i)$-th MP-controller's objective is to minimize the quadratic cost in (24), while the (i)-th linearized system is within $\Sigma$. Special care must be provided in order to correctly tune the prediction $N_{p}$ 
and control $N_{c}$ horizons. A long prediction horizon increases the predictive ability of the MP-controller but on the contrary it decreases the performance and demands more computations.

Each model predictive controller $V_{M P C}^{i}$ corresponds to the $i$-th error dynamic model of the articulated vehicle, obtained by solving the following optimization problem $\min J(k)$ with respect to the control moves variations $\Delta u$ and the error coordinates, while the discrete time sample index and $J(k)$ defined as:

$$
\begin{aligned}
J(k)= & \sum_{n=N_{W}}^{N_{p}}[\hat{\mathbf{y}}(k+n \mid k)-\mathbf{r}(k+n \mid k)]^{T} \mathbf{Q}[\hat{\mathbf{y}}(k+n \mid k)-\mathbf{r}(k+n \mid k)]+ \\
& +\sum_{n=0}^{N_{c}-1}\left[\Delta u^{T}(k+n \mid k) \mathbf{R} \Delta u(k+n \mid k)\right] \\
& +\sum_{n=N_{W}}^{N_{p}}[u(k+n \mid k)-\mathbf{s}(k+n \mid k)]^{T} \cdot \mathbf{N}[u(k+n \mid k)-\mathbf{s}(k+n \mid k)]
\end{aligned}
$$

where, $n$ is the index along the prediction horizon, $N_{w}$ is the beginning of the prediction horizon, $\mathbf{Q}$ is the output error weight matrix, $\mathbf{R}$ is the rate of change in control weight matrix, $\mathbf{N}$ is the control action error weight matrix, $\hat{\mathbf{y}}(k+n \mid k)$ is the predicted system's output at time $k+n$, given all measurements up to including those at time $k, \mathbf{r}(k+n \mid k)$ is the output set-point profile at time $k+n$, given all measurements up to including those at time $k$, $\Delta u(k+n \mid k)$ is the predicted rate of change in control action at time $k+n$, given all measurements up to including those at time $k, u(k+n \mid k)$ is the predicted optimal control action at time $k+n$, given all measurements up to and including those at time $k$, and $\mathbf{s}(k+n \mid k)$ is the input setpoint profile at time $k+n$, given all measurements up to and including those at time $k$.

Once all $V_{M P C}^{i}(k)$ controllers are computed, the objective of the $i \in \mathscr{S}$ controller is to stabilize the $i$-th system, while if $s$ increases, then the approximation of the error dynamic modeling is more accurate for a larger part of speed and slip angle and thus allowing the development of more efficient control algorithms.

\section{Simulation Results}

In the presented simulation results it is being assumed that direct measurements for both the velocity and the position of the vehicle can be performed, so no estimation action should be applied to the system, although in a real-life experimental setup, estimation of the slip angles should be performed, by utilizing one estimation algorithm (e.g. Extended Kalman filter).

For simulating the efficacy of the proposed control scheme for the problem of path following for an articulated vehicle, over a terrain with varying speed and slip angles, the following vehicle's characteristics have been considered: $l_{f}=0.6 \mathrm{~m}, l_{r}=0.8 \mathrm{~m}$. Twelve operating sets for the slip angle $\beta$ have been defined, in combination with three different vehicle's velocities (1, 2 and $3 \mathrm{~m} / \mathrm{sec}$ ), as it has been depicted in Table 1 .

As a result the overall lookup table that rules the switching signal for defining the $j$ active MPC control
Table 1. Active Regions for the Switching MPC

\begin{tabular}{|l|l|l|}
\hline MPC No. & Velocity $(\mathrm{m} / \mathrm{s})$ & Front slip $(\mathrm{rad})$ \\
\hline 1 & $0 \leq v<1$ & $0.00 \leq \beta<0.02$ \\
\hline 2 & $0 \leq v<1$ & $0.02 \leq \beta<0.04$ \\
\hline 3 & $0 \leq v<1$ & $0.04 \leq \beta<0.06$ \\
\hline 4 & $0 \leq v<1$ & $0.06 \leq \beta<0.08$ \\
\hline 5 & $1 \leq v<2$ & $0.00 \leq \beta<0.03$ \\
\hline 6 & $1 \leq v<2$ & $0.03 \leq \beta<0.05$ \\
\hline 7 & $1 \leq v<2$ & $0.05 \leq \beta<0.07$ \\
\hline 8 & $1 \leq v<2$ & $0.07 \leq \beta<0.09$ \\
\hline 9 & $2 \leq v \leq 3$ & $0.00 \leq \beta<0.04$ \\
\hline 10 & $2 \leq v \leq 3$ & $0.04 \leq \beta<0.08$ \\
\hline 11 & $2 \leq v \leq 3$ & $0.08 \leq \beta<0.12$ \\
\hline 12 & $2 \leq v \leq 3$ & $0.12 \leq \beta<0.16$ \\
\hline
\end{tabular}

( $s=12)$ is presented in Figure 4, while the total switching $\mathrm{MP}-$ controller is constructed by implementing a switching among the difference controllers in relation with the values of slip angle $\beta$ and the velocity $v$ respectively.

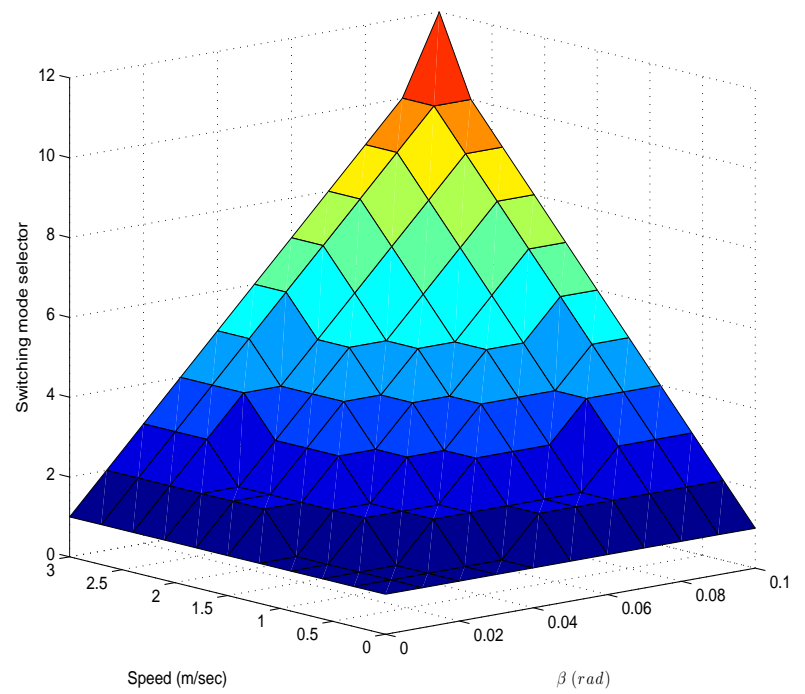

\section{Figure 4. Switching mode selector for MP- controllers}

The constraints imposed on the input and output variables, has been defined as it follows: the bounds on the articulated angle have been defined as $-0.785 \leq \gamma \leq 0.785$ (rad), and the bounds on the error dynamics has been set for the displacement error $-0.2 \leq e_{d} \leq 0.2$. The parameters for the twelve MP-controllers for the state and control action weighings matrices have been fine tuned separately according to the current active state space model. Moreover, in the simulation studies, correlation between the articulated angle $\gamma$ and the $\alpha$ slip angle, has been considered (derived from the geometry of the vehicle), denoted as: $\alpha=\arcsin \left(\frac{-l_{r}}{l_{f}+l_{r}} \sin \gamma\right)$. 
The cost function definition provides several crucial pieces of information, which are: length of the prediction horizon, variables included in this cost function and also the weights applied to each of the two components of the cost function. In the presented simulation results, the prediction horizon has been set to $N_{p}=10$ and the control horizon to $N_{c}=5$, with a control interval $(0.2 \mathrm{sec})$ for the all controllers.

The effectiveness of the proposed switching MPC scheme will be evaluated on tracking a circular reference path, while the significance and the important deterioration of the vehicle's response, under slip angles, has been already presented in [9]. In the presented simulations, a comparison will be performed between closed loop path tracking results, obtained from the proposed switching MPC scheme and the results obtained by having only one MPC fine tuned controller, under the existence of varying velocities and slip angles, as it is being presented in Figure 5.

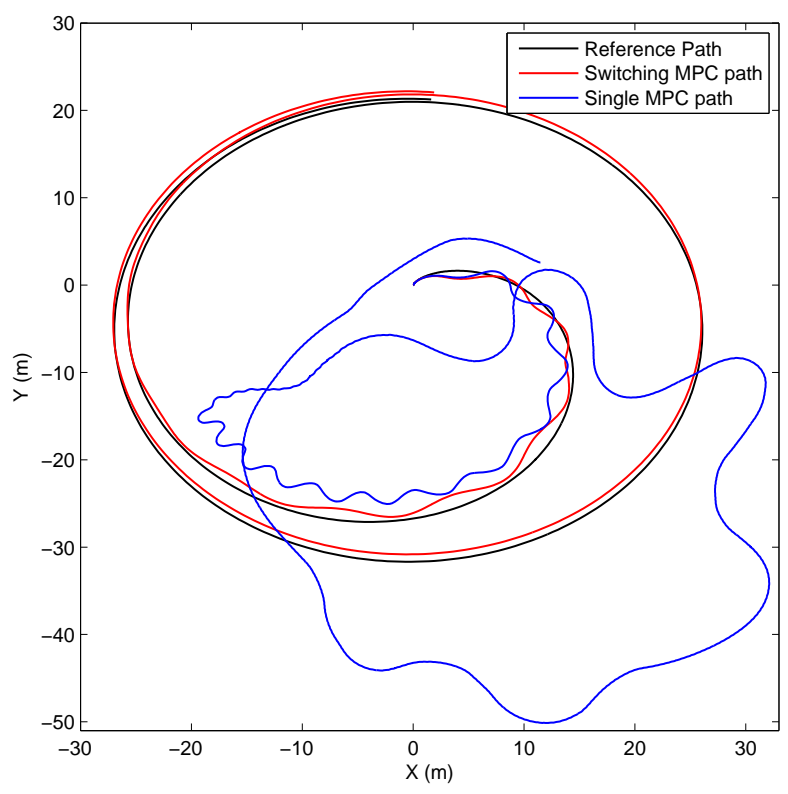

Figure 5. Circle path following based on switching MP-controller and single MPcontroller under varying velocities slip angles

From Figure 5 it is clear that the proposed switching MPC has the merit of adapting to the varying type of velocity and articulated angle and achieve a good path tracking performance, while the utilization of only one fixed MP-controller fails to track the circular reference path. In this simulation result, the fixed $2^{\text {nd }} \mathrm{MP}-$ controller has been utilized, as this is the controller that had the best simulation output under constant speed $(1 \mathrm{~m} / \mathrm{sec})$ and varying slip angles, while the utilization of different fixed type controllers has resulted in a system instability.
In Figure 6 the time evolution of the error corresponding dynamics, $\left(e_{d}, e_{h}\right.$ and $\left.e_{c}\right)$, from the path tracking response in Figure 5 is being presented, where it can be also observed that there is a significant error dynamic state tracking, for the case that the fixed MP-controller is being selected.
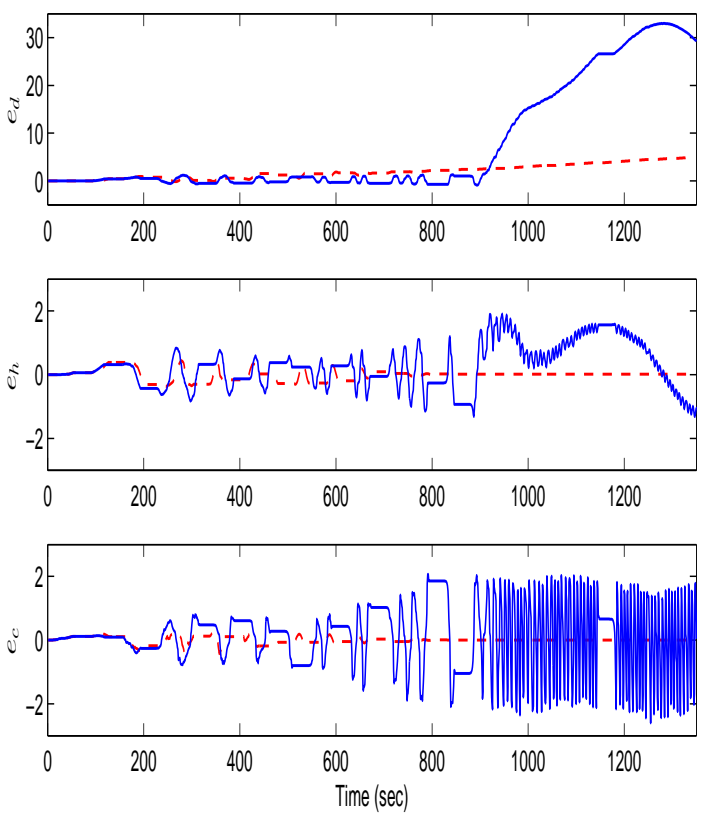

Figure 6. Time evolution of the system's
states (error dynamics) with switching MP-
controller and single MP-controller

In Figure 7 the corresponding responses for the simulated time varying velocity, and slip angle, for the path tracking response in Figure 5 are also being presented, with respect to the resulting mode selector signal, from Figure 4.

In addition in this figure, the response of the control effort (rate of the articulated angle $\dot{\gamma}$ and the pure response of the articulated angle is being also depicted. From the obtained simulation results, the superiority of the proposed switching MPC scheme is clear as it is able to achieve a good tracking of the reference path, under varying velocities and slip angles, a problem that it is highly linked to the real-life application. Finally, it should be noted that: a) in the presented simulation results, harsh switching and big slippage angles have been considered, that simulate the translation of the vehicle under heavy road conditions, and b) the same switching angles have been utilized as the testing scenario in all the depicted simulation results (single and switching $\mathrm{MP}$-controller). 


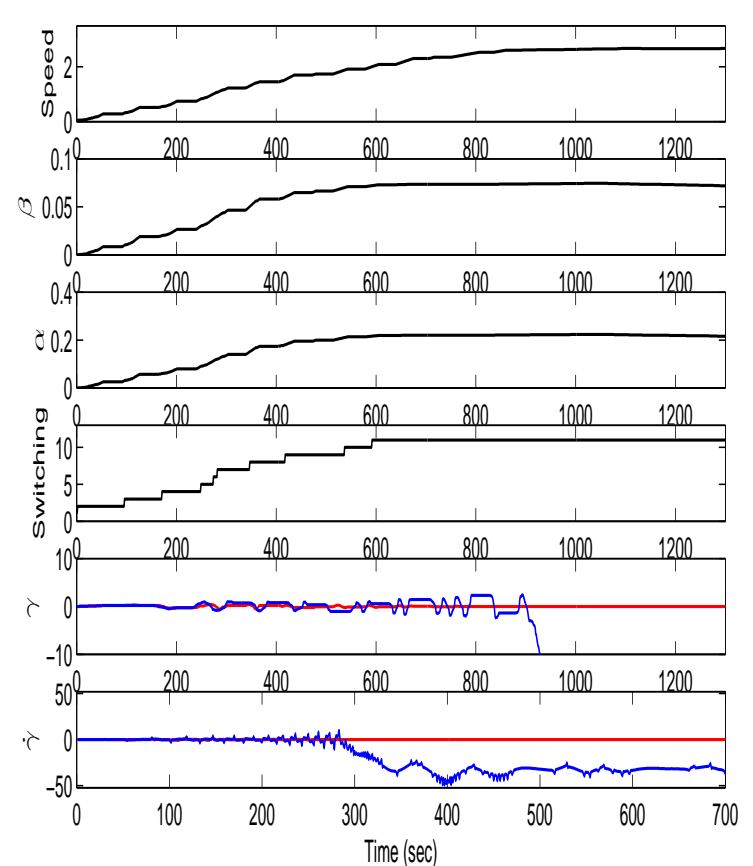

Figure 7. Corresponding varying velocity, slip angle, mode selector signal and control effort for switching and single MP-control

\section{Conclusions}

In this article a switching model predictive control scheme for an articulated vehicle under varying slip angles and different velocities has been presented. For the nonholonomic articulated vehicle, the non-linear kinematic model that is able to take under consideration the effect of the slip angles was extracted and been transformed into an error dynamics model. Based on the derived multiple error dynamic models, the varying slip angle has been considered as the switching rule and a corresponding switching mode predictive control scheme was designed. Simulation results have been presented that prove the efficacy of the overall suggested scheme.

\section{References}

[1] C. Altafini. A path tracking criterion for an lhd articulated vehicle. In International Journal of Robotics Research, May 1999.

[2] P. Bigras, P. Petrov, and T. Wong. A LMI approach to feedback path control for an articulated mining vehicle. In Electronics Research, 2002.

[3] P. Bolzern and A. Locatelli. A comparative study of different solutions to the path-tracking problem for an articulated vehicle. In Conference on Control Applications, pages 18-20, Glasgow, Scotland, U.K, 2002.
[4] R. M. Densantis. Modeling and path tracking for a load haul dump mining vehiclel. Transaction of the ASME, 119, 1997.

[5] A. Hemami and V. Polotski. Path tracking control problem formulation of an LHD loader. International Journal of Robotics Research, 18(5), 1999.

[6] F. Kuhne, J. Manoel, and W. F. Lages. Mobile robot trajectory tracking using model predictive control. In IEEE LARS, 2005.

[7] U. Larsson, G. Zell, K. Hyyppa, and A. Wernesson. Navigating an articulated vehicle and reversing with a trailer. In IEEE, 1994.

[8] J. Markdahl, G. Bortolin, and U. Andersson. Traction control for articulated off-road vehicle. In Master Thesis, KTH Royal Institute of Technology, 2010.

[9] T. Nayl, G. Nikolakopouls, and T. Gustafsson. Kinematic modeling and extended simulation studies of a load hull dumping vehicle under the presence of slip angles. In IASTED International Conference on Modelling, Simulation and Identification, pages 344-349, Pittsburgh, USA, 2011.

[10] F. Nunez, S. Navarro, A. Aguado, and A. Cipriano. State estimation based model predictive control for LHD vehicle. In 17th World Congress, the International Federation of Automatic Control, Seoul, Korea, 2008.

[11] P. Petrov and P. Bigras. A practical approach to feedback path control for an articulated mining vehicle. In Pros. of the 2001 IEEE/RSJ, Int. Conf. on Intelligent Robotics and Systems, aui, Hawaii, USA, Oct. 2001.

[12] D. Piyabongkarn, R. Rajamani, J. Grogg, and J. Lew. Development and experimental evaluation of a slip angle estimator for vehicle stability control. IEEE Trans on Control System Technology, 17(1):78-88, January 2009.

[13] P. Ridley and P. Corke. Autonomous control of an underground mining vehicle. In proc. Australian Conference on Robotics and Automation, Sydney, Nov. 2001.

[14] P. Ridley and P. Corke. Load haul dump vehicle kinematic and control. In Transaction of the ASME, 2003.

[15] S. Scheding, G. issanayake, E. Nebot, and H. Durrant. Slip Modeling and Aided Inertial Navigation of an LHD. In International conference on robotics and automation, New Mexico, 1997. 